\title{
Bilan des études de thermohydraulique des pompes primaires de réacteurs PWR
}

\author{
The results of studies of the thermohydraulics \\ of the primary pumps in $P W R$ reactors
}

\author{
P. Grison et J. F. Lauro
}

E.D.F. - Direction des Etudes et Recherches, Chatou

\section{Introduction}

Le développement des réacteurs nucléaires à eau pressurisée impose des normes très strictes pour assurer la sureté de la Centrale. L'ensemble du circuit primaire est ainsi dimensionné pour résister aux conséquences d'un accident grave, tel que l'accident de perte de réfrigérant primaire (APRP) par ouverture d'une brèche sur le circuit. L'analyse de 'sureté, qui est conduite pour l'étude de tels accidents, montre que les tacteurs déterminants sont :

- la température maximale dans les gaines,

- la surpression dans l'enceinte,

- les contraintes imposées aux matériels.

De plus, les différentes études paramétriques effectuées (voir par ex. réf. [1]) montrent que le comportement de la pompe joue un rôle essentiel dans la détermination de ces facteurs. En effet la pompe,

- soit joue le rôle de limiteur de débit lorsqu'elle se trouve sur la branche rompue,

- soit conditionne la répartition des débits dans le coeur lorsqu'elle est sur la branche intacte.

L'appréciation des marges de sureté, dont on dispose sur de tels réacteurs, nécessite donc une bonne connaissance du comportement de la pompe dans des situations accidentelles avec passage en diphasique. C'est dans ce but qu'E.D.F. a lancé en 1976 le programme EPOPEE (Essais de POmpes Primaires en Emulsion Eau-vapeur) de recherches sur ce sujet. Une campagne expérimentale, ainsi qu'une analyse théorique ont ainsi abouti en 1981 à une modélisation de la pompe en situation accidentelle. Nous présentons ici la synthèse de cette étude.

\section{Comportement de la pompe lors d'un APRP}

Afin de délimiter le domaine d'étude, une analyse globale peut permettre de prévoir les zones de fonctionnement possible de la pompe au cours d'un APRP.
La nupture peut se situer soit à l'amont, soit à l'aval de la pompe. Cependant dans les deux cas, l'eau pressurisée sortira par les deux extrémités de la branche rompue car la hauteur de refoulement de la pompe est bien inférieure à la différence de pression existant entre le circuit primaire et l'atmosphère. L'eau du circuit primaire va alors se vaporiser aux points d'étranglement du circuit. Le débit augmentera rapidement jusqu'à atteindre une valeur maximale, constituant le débit de blocage pour ce point.
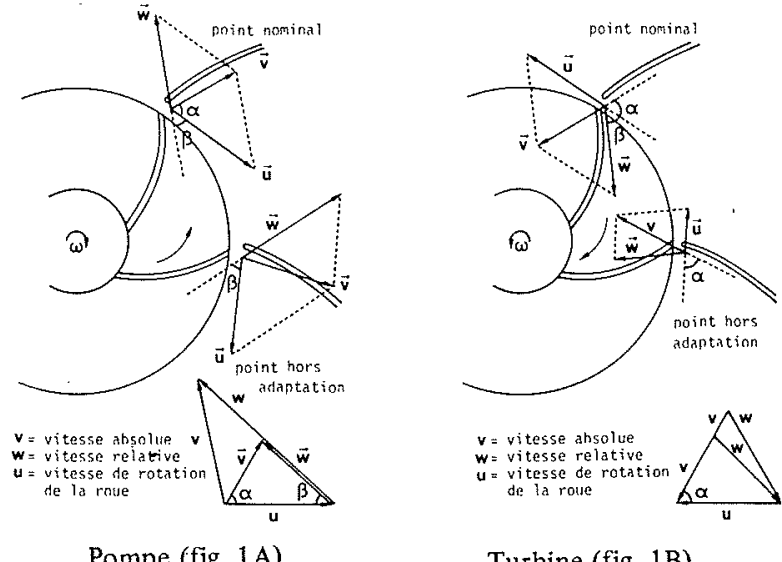

Turbine (fig. 1B)

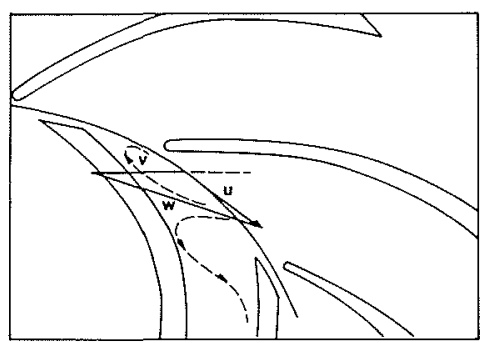

Construction du triangle des vitesses dans le $2^{\mathrm{e}}$ quadrant

Figure 1. - Fonctionnement à débit très désadapté. Analyse de l'angle et de la forme des filets fluides. 
Dans le cas d'une brèche amont, le débit au travers de la pompe va rapidement s'inverser. En raison de l'inertie de la pompe, et pour un court laps de temps, la pompe tournera toujours dans le sens initial, elle fonctionnera donc dans le deuxième quadrant. Si le système d'anti-dévirage ne fonctionne pas, la rotation de la pompe va s'inverser et conduire ensuite à un fonctionnement dans le troisième quadrant (rotation négative, débit négatif) avec deux zones de fonctionnement possibles : dissipation d'énergie ou turbine centripète.

Dans le cas d'une rupture aval, le débit ne change pas de sens mais au contraire augmente en accélérant la pompe. On passe donc dans la zone de turbine centrifuge du premier quadrant. La pompe va alors atteindre une survitesse dans le sens positif.

Les pompes dans les branches intactes sont elles aussi soumises à des débits positifs ou négatifs, avec des conditions d'entrée très diverses : eau, eau + vapeur ou vapeur. Mais les conditions seront beaucoup moins sévères que pour la pompe de la branche rompue. Le modèle mathématique retenu devra cependant être capable de prévoir aussi leur comportement afin d'avoir une description complète de la distribution du débit dans ces branches.

On retiendra ainsi comme domaine d'étude :

\section{- débit positif rotation positive}

l'ensemble du domaine (limité dans la pratique à 2,5 fois la vitesse de rotation nominale $\omega_{0}$ )

- débit négatif rotation positive pour les vitesses $\leqslant \omega_{0}$ - débit négatif rotation négative l'ensemble du domaine (voir réf. [22] "Définition du domaine d'essais")

\section{Analyse des phénomènes se produisant dans une pompe au cours d'un APRP}

\subsection{Fonctionnement à régime très désadapté}

Nous avons vu que le débit traversant la pompe pouvait varier énormément, allant du débit négatif au surdébit positif en passant par le débit nul. Nous devrons donc avoir une représentation adaptée à ces conditions, très éloignées des conditions normales. I] est alors très important de connaître la forme des filets fluides à l'intérieur de la pompe.

L'étude détaillée de ces phénomènes a été faite dans la réf. [2].Elle s'appuie sur une analyse théorique et sur une visualisation faite à partir d'une maquette bidimensionnelle. Nous ne donnerons ici que les principaux résultats.

$L$ hypothèse de base est qu'il y a une continuité de la vitesse absolue entre les parties fixes et les parties mobiles. On obtiendra donc la vitesse dans un repère (fixe ou mobile), connaissant celle dans l'autre repère, par la construction du triangle des vitesses. Ceci revient à dire que l'écoulement garde la direction imposée par l'élément amont. (fig. $1 \mathrm{a}-1 \mathrm{~b}-1 \mathrm{c}$ ).

Conséquences : les principales conséquences de cette modélisation seront :

- la détermination de la "hauteur" théorique de pompe qui découle alors directement des modules et des directions des vitesses entrée/sortie roue par l'équation d'Euler;
- une géométrie fictive "variable" qui s'adaptera aux formes des filets fluides;

- l'existence de termes de pertes pour tenir compte de la désadaptation (par exemple dans le $2^{\mathrm{e}}$ quadrant toute l'énergie cinétique relative est dissipée puisque $l^{\prime}$ incidence est proche de $90^{\circ}$ ). De plus, des termes de pertes sont introduits aux faibles débits pour tenir compte d'une inhomogénéité de l'angle $\beta$ ce qui entraine de fortes recirculations dans la roue.

\subsection{Changement de phase dans la roue}

On montre, à partir de l'étude de la croissance d'une bulle isolée, que le taux de vaporisation (ou de condensation) est limité par des phénomènes thermiques (voir réf. [3]), pour les températures rencontrées dans le circuit primaire. Macroscopiquement on introduit donc un temps de relaxation $\theta$ limitant ce changement de phase.

L'expérience montre que $\theta$ est inférieur au temps de passage dans la roue. Il y aura donc changement de phase dans la roue. Les caractéristiques de la pompe ne dépendent donc plus seulement du champ de vitesse à l'entrée de la pompe, car un changement d'état dans la roue donnera un débit volumique entrée très différent d'un débit volumique sortie et les triangles de vitesse entrée et sortie seront donc également très différents. Ceci est bien mis en évidence expérimentalement sur la courbe d'évolution du coiple lors d'une dépressurisation : on constate en effet, à partir d'une certaine valeur de la pression aval, que le débit n'augmente pratiquement plus, par contre le couple, lui, continue à évoluer, ce qui prouve une vaporisation à l'intérieur de la roue (voir fig. 5).

Le débit limite calculé en déséquilibre sera donc très différent de celui calculé à l'équilibre. Signalons de plus, qu'en diphasique, on se situe très souvent dans une zone appelée zone de pseudo-blocage, zone où la vaporisation est très intense (voir par ex. réf. [4]).

\subsection{Glissement entre phases}

Dans un écoulement à deux phases, la vitesse de chaque phase sera déterminée par : (voir réf. [3])

- le gradient de pression;

- l'inertie de chaque phase ;

- les échanges entre phases:

- échange de masse

- frottement interfacial

- masse induite

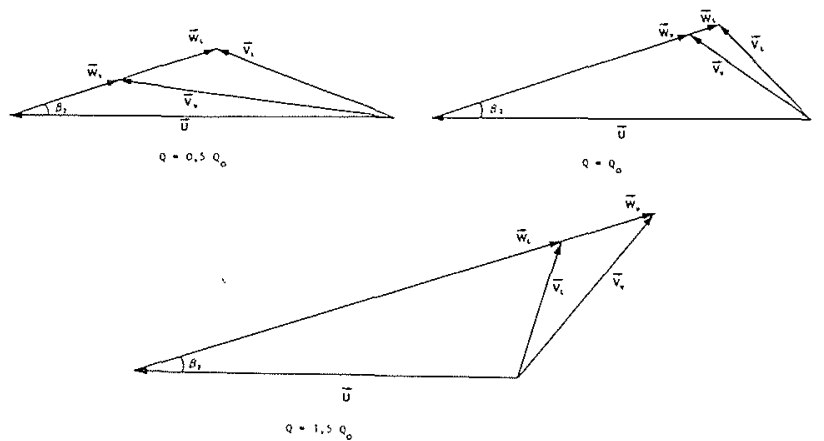

Figure 2 - Evolution des triangles des vitesses en sortie de roue pour différents débits d'entrée. $(P=60$ bars, taux de vide entrée $=0,5$ ) . 


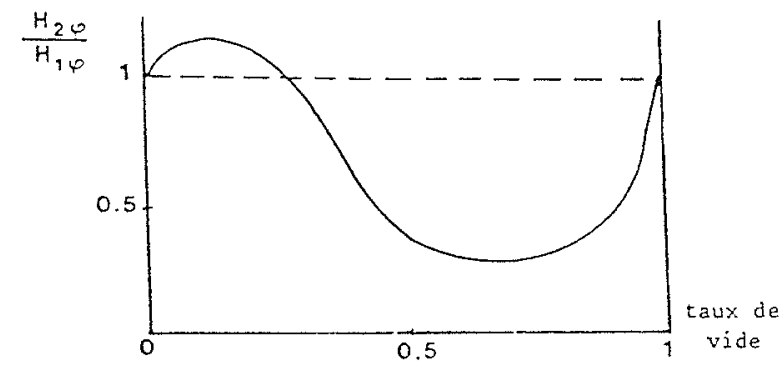

Figure 3 - Coefficient de dégradation en supposant un glissement entre phases.

- le frottement à la paroi ;

- les forces extérieures éventuelles.

En particulier, dans un écoulement accéléré ou décéléré les deux phases auront des vitesses très différentes.

Dans le cas de la pompe, les vitesses en sortie de roue peuvent donc être très différentes, ce qui modifiera le triangle des vitesses en sortie de roue. L'équation d'Euler devra alors être écrite pour chacune des phases, ce qui modifiera donc la "hauteur" de la pompe. La figure 2 représente ainsi l'évolution de ces triangles de vitesses pour le premier quadrant.

On montre ainsi qu'il existe une "dégradation" des courbes hauteur/débit par rapport au cas monophasique (fig. 3).

Une étude paramétrique montre (voir réf. [9]) que ce phénomène est d'autant plus amplifié que la pression est faible. Il est donc très important de bien le représenter pour simuler les essais à faible pression. Notons également que ces courbes ont été obtenues avec des coefficients de frottement interfaciaux obtenus sur des géométries fixes. Nous n'avons pas cherché à l'ajuster sur les essais pompe. En particulier s'il y a stratification dans la pompe (voir paragraphe suivant) il faut certainement dimininuer ce coefficient.

\subsection{Autres phénomènes}

D'autres phénomènes peuvent modifier sensiblement le comportement de la pompe. Citons en particulier :

- la prérotation;

- la post-rotation en débit inverse ;

- la stratification des deux phases dans la roue.

Ces phénomènes sont pris en compte dans le modèle sous forme de pertes supplémentaires (pour plus de détails, voir réf. [9]).

\section{Modélisation de la pompe en diphasique}

Le modèle retenu pour la description de la pompe devra pouvoir prendre en compte les différentes phénomènes décrits dans la partie précédente. Ces principales caractéristiques seront alors :

(on trouvera une description plus complète dans la réf. [9])

Modèle axial afin de tenir compte des changements de phase intervenant dans la roue. On aura donc une description successive de l'aspiration, de la roue, du diffuseur et de la volute. De plus ce modèle sera à "géométrie variable" afin de prendre en compte la modification des filets fluides.

Modèle basé sur la théorie d'Euler avec adjonction de termes de pertes.

Modèle à retard thermodynamique : le débit de blocage est alors une conséquence directe du calcul et ne nécessite aucune corrélation supplémentaire.

Modèle à glissement de phases afin d'obtenir une bonne description du phénomène de dégradation des caractéristiques.

Les principales équations du modèle seront alors :

masse $: \frac{d}{d Z}\left(A \alpha_{K}, \rho_{K}\right)=A \Gamma$

avec

$\Gamma=$ échange de masse entre phases

impulsion $\frac{d}{d Z} A \alpha_{K}\left(\rho_{K} V_{K}^{2}+P\right)+\frac{1}{2} A \alpha_{K}\left(1-\alpha_{K}\right) \rho_{m}\left(V_{K} d \frac{V_{K}}{d z}-V_{K^{\prime}} \frac{\left.d V_{K^{\prime}}\right)}{d z}\right) \underbrace{-A\left(P-p_{i}\right) \frac{d \alpha_{K}}{d z}}_{\text {masse induite }}$

$$
\begin{gathered}
=A I-\chi_{K} \zeta_{K}+A \alpha_{K} \rho_{K} g z+\alpha\left(P-p_{i}\right) \frac{d A}{d z} \\
\begin{array}{c}
\text { échange frottement accélération } \\
\text { d'impulsion en paroi i } \\
\text { interfacial }
\end{array}
\end{gathered}
$$$$
\text { énergie } \frac{d}{d z} A \alpha_{K} \rho_{K} V_{K}\left(h_{K}+\frac{V_{K}^{2}}{2}\right)+\frac{1}{2} A \alpha_{K}\left(1-\alpha_{K}\right) \rho_{m}\left(V_{K} \frac{d V_{K}}{d z}-V_{K^{\prime \prime}} \frac{d V_{K^{\prime}}}{d z}\right) \underset{\text { vitesse }}{W i}
$$$$
\text { masse induite }
$$

$$
\begin{array}{cc}
=A Q+ & A \alpha_{K} \rho_{K} g_{z} V_{K} \\
\begin{array}{c}
\text { apport } \\
\text { de claleur }
\end{array} & \begin{array}{c}
\text { accélération } \\
\text { axiale }
\end{array}
\end{array}
$$


avec

pour la vapeur $\quad K=$ vapeur et $K^{\prime}=$ liquide

pour le liquide $K=$ liquide et $K^{\prime}=$ vapeur

$g_{z}=0$ dans les parties fixes

$g_{z}=\frac{1}{2} \omega^{2} d R^{2} / d z$ dans la roue

Le modèle introduit ici semble donc relativement complexe car il essaye de décrire les différents phénomènes de manière aussi complète que possible. Le temps de calcul sera donc plus élevé que dans les autres descriptions. Par contre, il utilise très peu de corrélations expérimentales, ce qui lui assure un domaine de validité relativement étendu et surtout une bonne fiabilité pour le transfert à l'échelle 1 .

\section{Programme expérimental}

Un important programme expérimental a été développé afin de valider le modèle théorique. Le Tableau I compare le domaine étudié aux autres programmes d'essais de pompe en écoulement diphasique (eau-vapeur).

\subsection{Description de la boucle expérimentale EPOPEE}

(On trouvera une description détaillée dans les réf. [14] et [15])

Présentation :

Un schéma de principe de la boucle est donné sur la figure 4. Elle se compose essentiellement :

- d'une chaudière à haute pression assurant la mise en condition du fluide ;

- d'un ensemble de deux bidons d'environ $7 \mathrm{~m}^{3}$ chacun, l'un étant rempli d'eau chaude pressurisée dans les conditions voulues pour l'essai, l'autre étant rempli de vapeur à une pression supérieure, son rôle étant de maintenir dans le premier bidon une pression constante pendant la durée de l'essai ;

- d'un jeu de différentes tuyauteries permettant la liaison entre B1 et la pompe. Des convergents divergents de tailles différentes permettent de fixer la détente entre B1 et la pompe, et de faire varier ainsi le taux de vide à l'entrée de la pompe ;

- de la maquette de pompe. C'est un modèle au $1 / 10^{\mathrm{e}}$ des pompes primaires. Son régime nominal est à $15000 \mathrm{tr} / \mathrm{min}$ et l'installation peut tourner $35000 \mathrm{tr} / \mathrm{min}$. Elle est entrainée par un ensemble Ward-Léonard de $2 \mathrm{MW}$ et une ligne d'arbre rapide. Elle peut se monter dans les deux sens ce qui permet d'avoir des débits positifs ou négatifs;

- d'une vanne de détente en sortie de pompe qui permet de régler la contrepression. Cette vanne est pourvue d'un automatisme permettant soit la régulation du débit, soit la programmation de séquences au cours de l'essai; - d'un silencieux récupérateur permettant le rejet à l'atmosphère.

Le mode de fonctionnement choisi est une succession de régimes permanents d'environ $20 \mathrm{~s}$. Des essais complémentaires en transitoires peuvent également être réalisés.

\subsection{Essais "Pompe diphasique"}

Ces essais ont permis de tester le comportement de la pompe en régime permanent dans les trois premiers quadrants.

\subsubsection{Essais effectués}

A. Domaine couvert pour le premier quadrant

Au cours de cette campagne, il a été effectué 32 rafales ce qui a permis d'obtenir 148 points d'essais.

\begin{tabular}{|l|c|c|}
\hline \multicolumn{2}{|c|}{ Domaine couvert lors de la première campagne d'essais } \\
\hline & Minimum & Maximum \\
\hline Pression d'entrée & $29 \mathrm{bars}$ & $113 \mathrm{bars}$ \\
Pression de sortie & $8 \mathrm{bars}$ & $120 \mathrm{bars}$ \\
Température d'entrée & $230^{\%} \mathrm{C}$ & $320 \% \mathrm{C}$ \\
Taux de vide : entrée & $0 \%$ & $80 \%$ \\
Taux de vide : sortie & $0 \%$ & $100 \%$ \\
Débit massique & $10 \mathrm{~kg} / \mathrm{s}$ & $75 \mathrm{~kg} / \mathrm{s}$ \\
Vitesse de la pompe & $7500 \mathrm{tr} / \mathrm{min}$ & $25000 \mathrm{tr} / \mathrm{min}$ \\
\hline
\end{tabular}

Tableau I - Tableau des principaux programmes d'essais de pompe en écoulement diphasique eau/vapeur.

\begin{tabular}{|c|c|c|c|c|c|c|}
\hline & échelle & $\begin{array}{l}\text { vitesse } \\
\text { nominale }\end{array}$ & $\begin{array}{l}\text { génēration du } \\
\text { diphasique }\end{array}$ & $\begin{array}{l}\text { nombre de } \\
\text { points }\end{array}$ & $P \max$ & Observations \\
\hline $\begin{array}{l}\text { Combustion } \\
\text { Engineering }\end{array}$ & $1 / 5$ & $4.500 \mathrm{c} / \mathrm{m}$ & $\begin{array}{l}\text { par mêlange } \\
\text { boucle fermée }\end{array}$ & $\begin{array}{c}1.000 \text { en perma- } \\
\text { nent } \\
16 \text { transi- } \\
\text { toires }\end{array}$ & 80 bars & $\begin{array}{l}\text { Donne de bonnes indications sur le com- } \\
\text { portement de la pompe, mais résultats } \\
\text { difficilement interprétables en détail } \\
\text { car il y a beaucoup d'incohérences entre } \\
\text { les mesures. }\end{array}$ \\
\hline $\begin{array}{l}\text { E.V.A. } \\
\text { C.E.A. - } \\
\text { WESTINGHOUSE }\end{array}$ & $1 / 3$ & $1.400 \mathrm{t} / \mathrm{mm}$ & $\begin{array}{l}\text { par mélangc } \\
\text { boucle fermée }\end{array}$ & 600 points & 30 bars & $\begin{array}{l}\text { Essais faits avec un } \Delta p \text { très faible, d'où } \\
\text { forte dispersion des rêsultats. } \\
\text { Résultats utiles surtout dans le ler } \\
\text { quadrant. }\end{array}$ \\
\hline $\begin{array}{l}\text { ATOMIC ENERCY } \\
\text { OF CANADA }\end{array}$ & $\begin{array}{l}\emptyset \text { roue }= \\
295 \text { mm. }\end{array}$ & $3.600 \mathrm{t} / \mathrm{m} \mathrm{n}$ & $\begin{array}{l}\text { par mélange } \\
\text { boucle fermée }\end{array}$ & & 55 bars & \\
\hline LPOPEE & $1 / 10$ & $15.000 \mathrm{t} / \mathrm{mn}$ & $\begin{array}{l}\text { auto-vaporisation } \\
\text { boucle ouverte }\end{array}$ & 700 points & 120 bars & $\begin{array}{l}\text { taux de vide entrée limité (autovapori- } \\
\text { sation) - Forts } \Delta \rho \text { dans la pompe, donc } \\
\text { nombre de points important avec débit } \\
\text { limite. }\end{array}$ \\
\hline
\end{tabular}




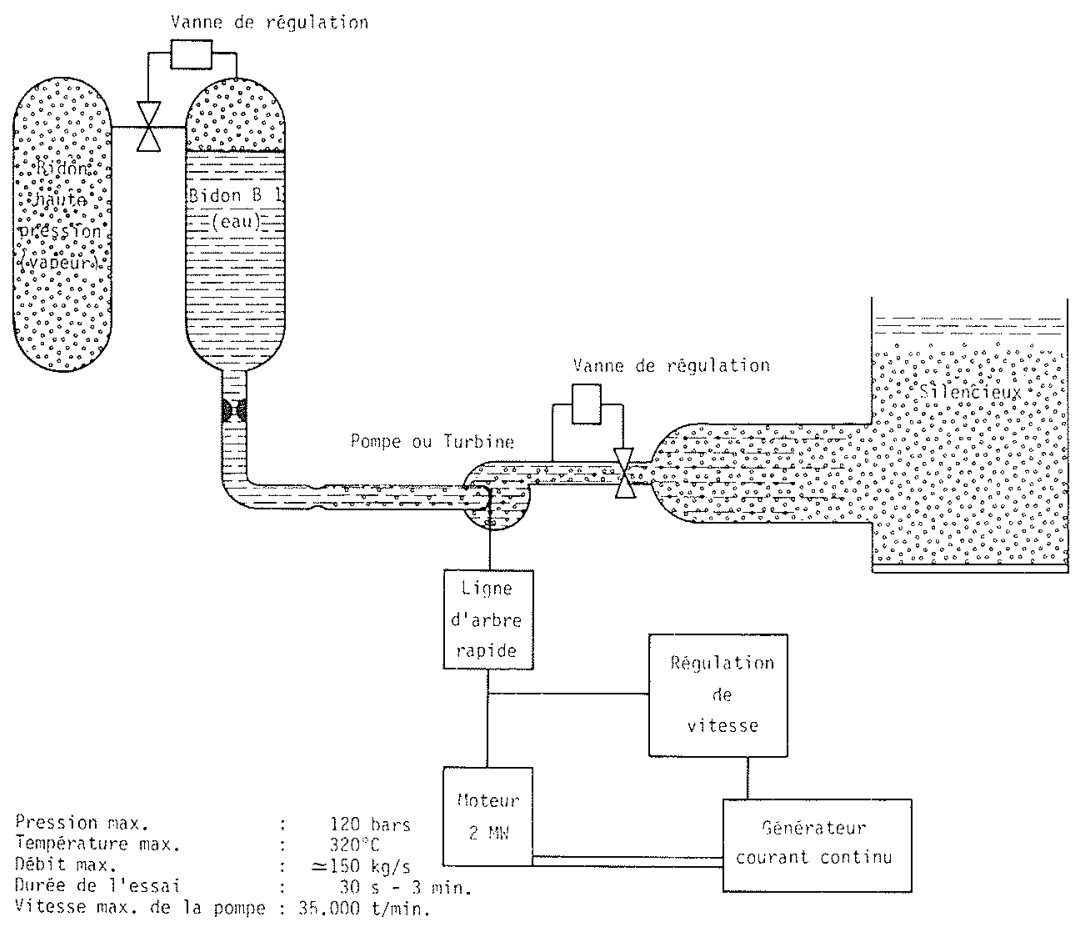

Figure 4 - Boucle expérimentale EPOPEE.

\section{B. Second quadrant}

Dans cette zone de fonctionnement à débits négatifs et rotation positive, 31 rafales ont été effectuées, ce qui a permis d'obtenir 238 points d'essais.

\begin{tabular}{|l|c|c|}
\hline \multicolumn{3}{|c|}{ Domaine couvert } \\
\hline & Minimum & Maximum \\
\hline Pression d'entrée & 33 bars & 114 bars \\
Pression de sortie & $7 \mathrm{bars}$ & $107 \mathrm{bars}$ \\
Température d'entrée & $239^{\circ} \mathrm{C}$ & $312^{\circ} \mathrm{C}$ \\
Taux de vide : entrée & $0 \%$ & $51 \%$ \\
Taux de vide : sortie & $0 \%$ & $98 \%$ \\
Débit massique & $4 \mathrm{~kg} / \mathrm{s}$ & $47 \mathrm{~kg} / \mathrm{s}$ \\
Vitesse de la pompe & $7500 \mathrm{tr} / \mathrm{min}$ & $15000 \mathrm{tr} / \mathrm{min}$ \\
\hline
\end{tabular}

\section{Troisième quadrant}

Dans cette zone de fonctionnement à débits négatifs et rotation négative, 43 rafales ont été effectués, ce qui a permis d'obtenir 268 points d'essais.

\begin{tabular}{|l|c|c|}
\hline \multicolumn{3}{|c|}{ Domaine couvert } \\
\hline & Minimum & Maximum \\
\hline Pression d'entrée & 26 bars & 108 bars \\
Pression de sortie & 5 hars & 113 bars \\
Température d'entrée & $229^{\circ} \mathrm{C}$ & $315^{\circ} \mathrm{C}$ \\
Taux de vide : entrée & $0 \%$ & $99 \%$ \\
Taux de vide : sortie & $0 \%$ & $99 \%$ \\
Débit massique & $10 \mathrm{~kg} / \mathrm{s}$ & $60 \mathrm{~kg} / \mathrm{s}$ \\
Vitesse de la pompe & $-7500 \mathrm{tr} / \mathrm{min}$ & $-32000 \mathrm{tr} / \mathrm{min}$ \\
\hline
\end{tabular}

\section{Rotor bloqué}

Dansircette zone de fonctionnement débits négatifs et vitesse de rotation nulle, 10 rafales ont été effectuées ce qui a permis d'obtenir 75 points d'essais.

\begin{tabular}{|l|c|c|}
\hline \multicolumn{3}{|c|}{ Domaine couvert } \\
\hline & Minimum & Maximum \\
\hline Pression d'entrée & 41 bars & 113 bars \\
Pression de sortie & 8 bars & $113 \mathrm{hars}$ \\
Température d'entrée & $259^{\circ} \mathrm{C}$ & $316^{\circ} \mathrm{C}$ \\
Taux de vide : entrée & $0 \%$ & $69 \%$ \\
Taux de vide : sortie & $0 \%$ & $99 \%$ \\
Débit massique & $8 \mathrm{~kg} / \mathrm{s}$ & $53 \mathrm{~kg} / \mathrm{s}$ \\
\hline
\end{tabular}

\subsubsection{Comportement de la pompe}

Les essais sont obtenus par diminution de la pression aval par palier, à partir d'un régime monophasique. I1 s'établit alors progressivement un régime de blocage dans la pompe. Sur les figures $5 a-5 b-5 c$ on peut voir l'évolution des différents paramètres au cours d'un essai dans chaque quadrant. On peut également voir une vaporisation progressive d'abord à l'amont de la pompe, puis dans la pompe. On observe également un blocage du débit alors que le couple continue à évoluer ce qui est caractéristique d'une vaporisation dans la roue.

\subsubsection{Remarque sur le glissement et le taux de vide}

Compte tenu de la grande sensibilité du calcul des caractéristiques de la pompe à la valeur du glissement, une attention particulière a été portée sur la détermination du taux de vide et sur l'évaluation du glissement en amont et en aval de la pompe.

On a donc comparé les résultats donnés par les deux mesures du taux de vide (gammagraphie et méthode de dilution) ainsi que la valeur calculée à partir du titre massique thermodynamique (voir réf. [18] pour les détails du calcul). On a pu ainsi s'assurer de la cohérence des résultats et dégager les tendances suivantes:

- A l'amont de la pompe, l'écoulement se présente 

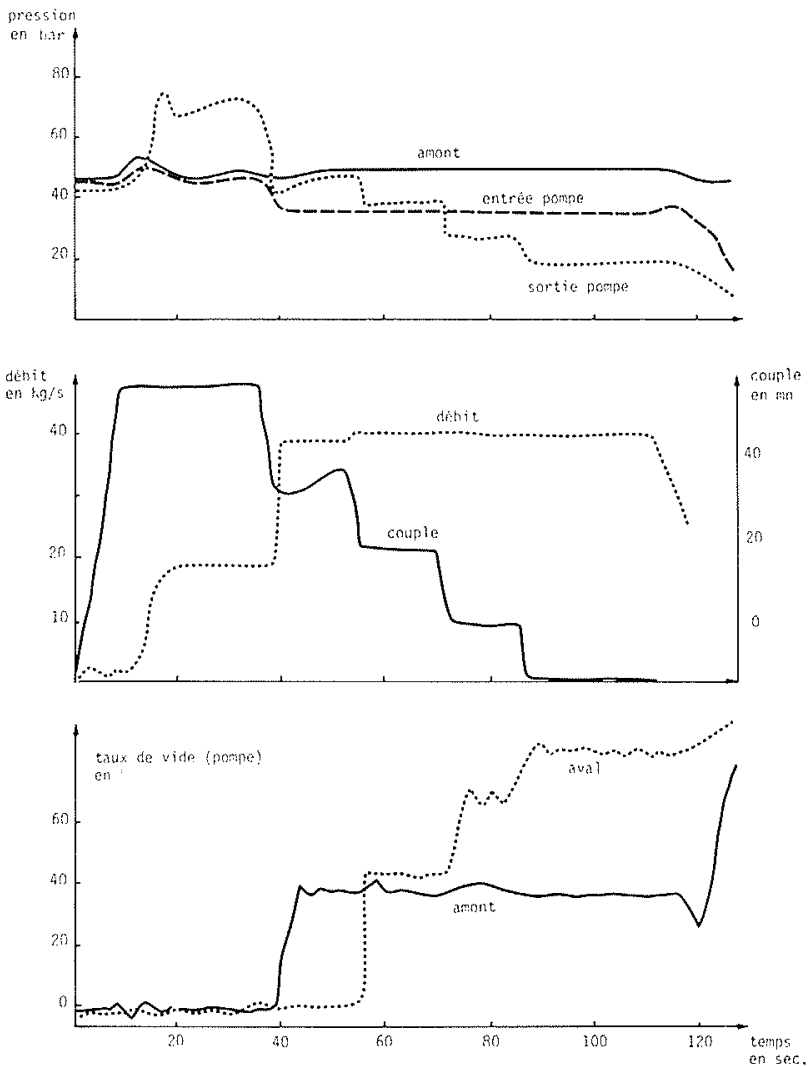

Figure 5A. - Evolution des différents paramètres au cours de l'essai vitesse de rotation : $25000 \mathrm{t} / \mathrm{mm}$.
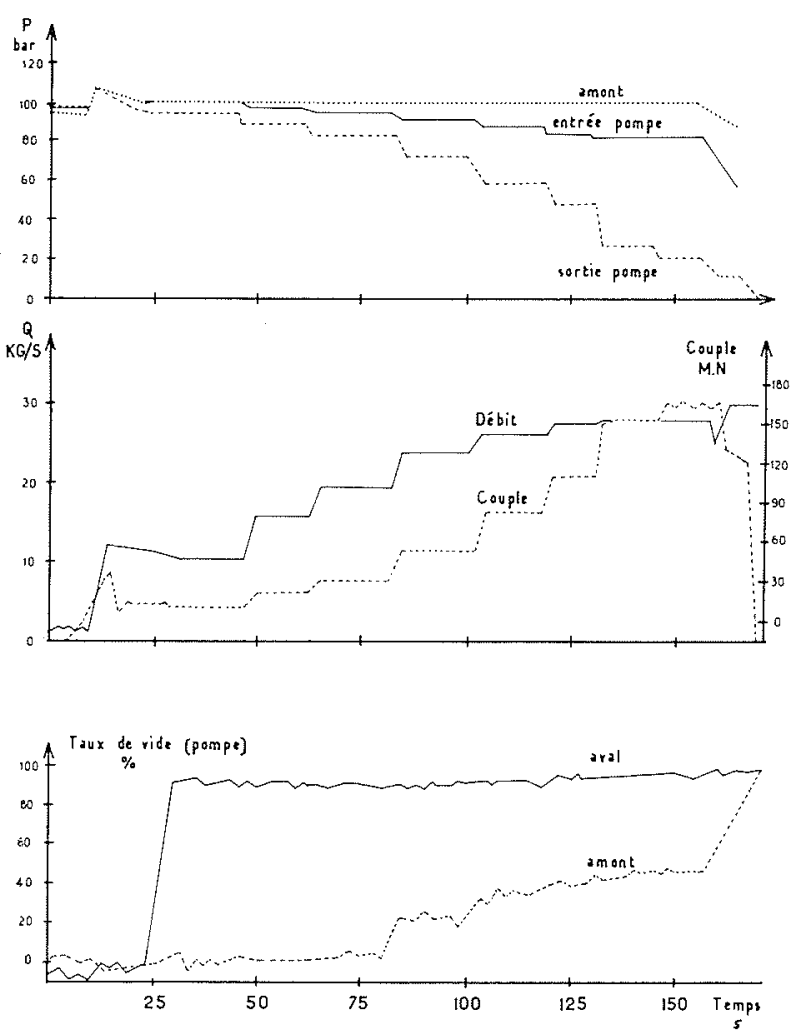

Figure 5B - Evolution des différents paramètres au cours de l'essai. Vitesse de rotation $11000 \mathrm{t} / \mathrm{mn}$. Deuxième quadrant.
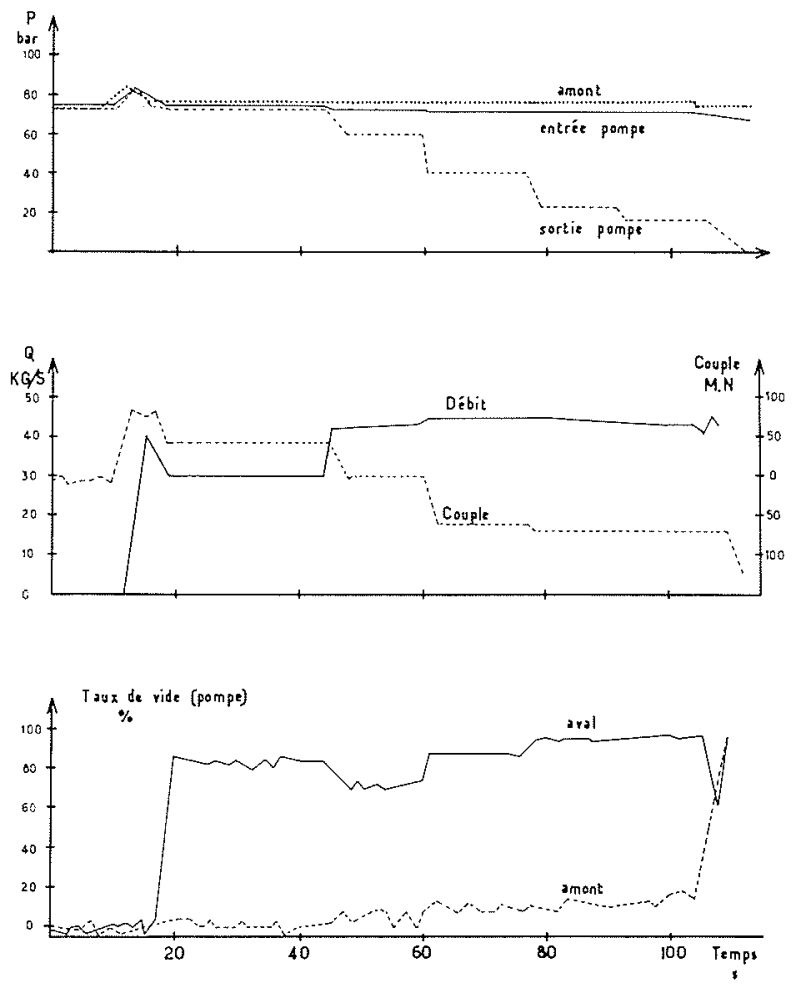

Figure $5 \mathrm{C}-$ Evolution des différents paramètres au cours de l'essai. Vitesse de rotation - $25000 \mathrm{t} / \mathrm{mn}$. Troisième quadrant.

presque toujours sous forme dispersée et homogène. Le glissement entre phases reste voisin de 1 ;

- A l'aval de la pompe, pour le premier quadrant l'écoulement semble également homogène avec un glissement proche de 1 , la dispersion autour de cette valeur est cependant plus grande que dans le cas précédent. Pour les deuxième et troisième quadrants l'écoulement est presque toujours annulaire avec un glissement variant entre 0,2 et 5 .

\section{Comparaison théorie-expérience - Résultats EPOPEE}

pour le $1^{\text {er }}$ quadrant $(Q>0, \omega<0)$

Les prévisions de débit critique sont relativement bonnes $(+0,+15 \%)$ pour l'ensemble des points. Le comportement de la pompe est bon et il faut souligner que le comportement du couple en régime pseudobloqué est bien retrouvé (variation du couple sans changement du débit à l'entrée de la pompe). Les prévisions du taux de vide sont également bonnes. Un exemple de résultat est donné sur la figure 6 .

pour le $3^{e}$ quadrant $(Q<0, \omega<0)$

Le comportement général de la pompe est bien prévu, et l'évolution du couple est bonne. Les prévisions du débit critique restent acceptables $(+5 \%,+30 \%)$. Cet écart, plus important que pour le $1^{\mathrm{er}}$ quadrant, s'explique par l'importance de la post-rotation qui engendre une stratification des deux phases, non prise en compte par le modèle. 


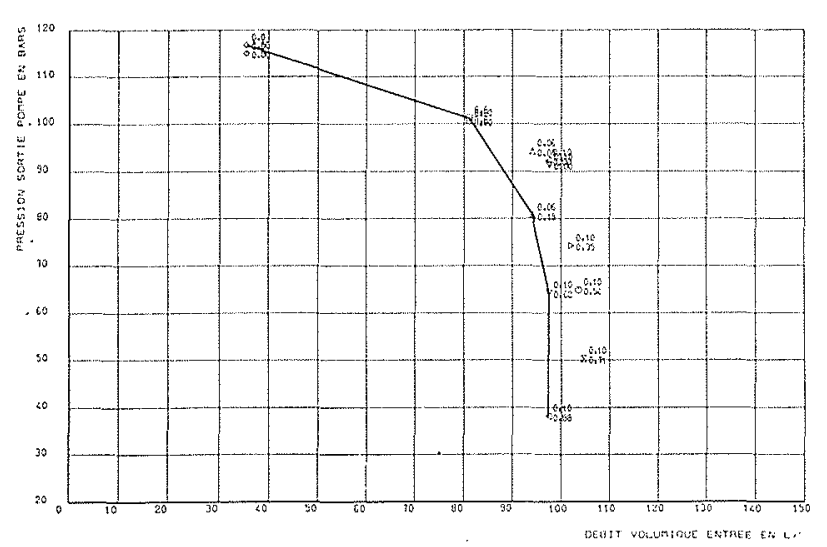

Figure 6A

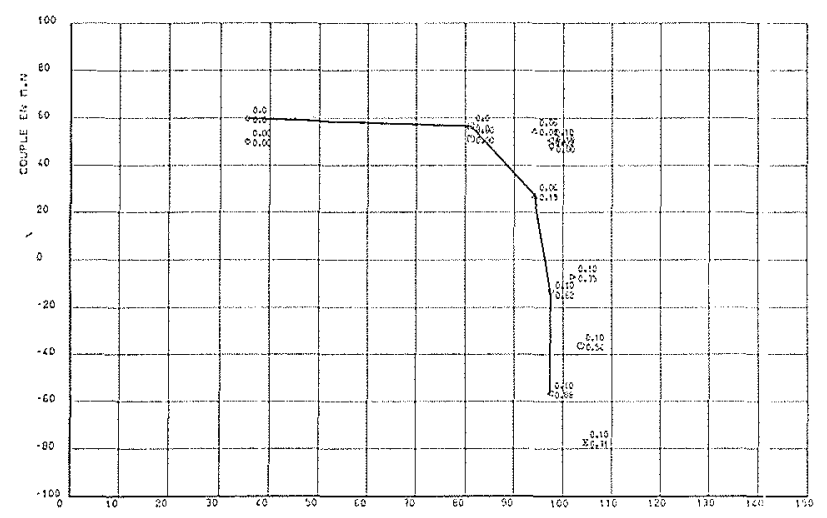

Figure 6B

\section{pour le $2^{e}$ quadrant}

La très forte dissipation d'énergie que l'on rencontre dans la roue pour ce régime rend le calcul très délicat et nous n'avons obtenu que des résultats partiels dans cette zone. Le débit critique calculé semble ici inférieur au débit expérimental.

\section{Exemple d'application : calcul de survitesse}

Le modèle décrit précédemment peut servir à la description du comportement du circuit primaire lors d'une ouverture de brèche. Nous n'examinons pas ici le comportement global du circuit primaire, cette description faisant intervenir tous les composants de ce circuit. Nous nous proposons seulement d'analyser le comportement de la pompe en supposant connues et constantes les conditions à l'amont de la pompe.

Dans le cas d'une rupture à l'aval de la pompe, nous avons déjà montré que le débit augmentait jusqu'à ce que les conditions critiques soient atteintes en un point du circuit. Le couple hydraulique peut alors atteindre des valeurs supérieures au couple maximum du moteur, il y a alors "décrochage" puis emballement de la pompe. De même il peut $\mathrm{y}$ avoir emballement en cas de perte d'alimentation électrique du moteur.

\section{Brèche aval - Pleine ouverture}

Nous avons fixé les conditions suivantes amont :

$$
P=60 \mathrm{~b} \quad \text { taux de vide } \alpha=0,5
$$

Le calcul montre alors qu'il s'établit un premier blocage en sortie de roue. Pour ce débit, le régime est loin d'être bloqué à la brèche, il s'établira donc une surdétente dans le diffuseur afin d'obtenir un nouveau blocage à la brèche. La figure 7 présente l'évolution du débit et du couple en fonction du $\Delta P$ aux borres de la pompe. Dans cette configuration le couple est de l'ordre de $10^{5} \mathrm{~m} . \mathrm{N}$., ce qui, compte tenu de l'inertie de la pompe, donne une accélération

$$
\frac{d \omega}{d t}=\frac{C}{I} \simeq 400(\mathrm{t} / \mathrm{min}) / \mathrm{s}
$$

La vitesse augmente alors rapidement, ce qui entraîne une augmentation du débit. On observera sur la figure 8 les différents profils de pression. Le calcul montre que le couple s'annule lorsque la vitesse est de l'ordre de $4200 \mathrm{tr} / \mathrm{min}$. Le débit est alors de $5400 \mathrm{~kg} / \mathrm{s}$.

\section{Brèche aval - section réduite à $50 \%$}

Nous gardons les mêmes conditions amont $(60 \mathrm{~b}$, $\alpha=0,5)$ mais la taille de la brèche est réduite à la moitié de la section de la tuyauterie. Nous observons alors que le blocage se produit directement à la brèche,

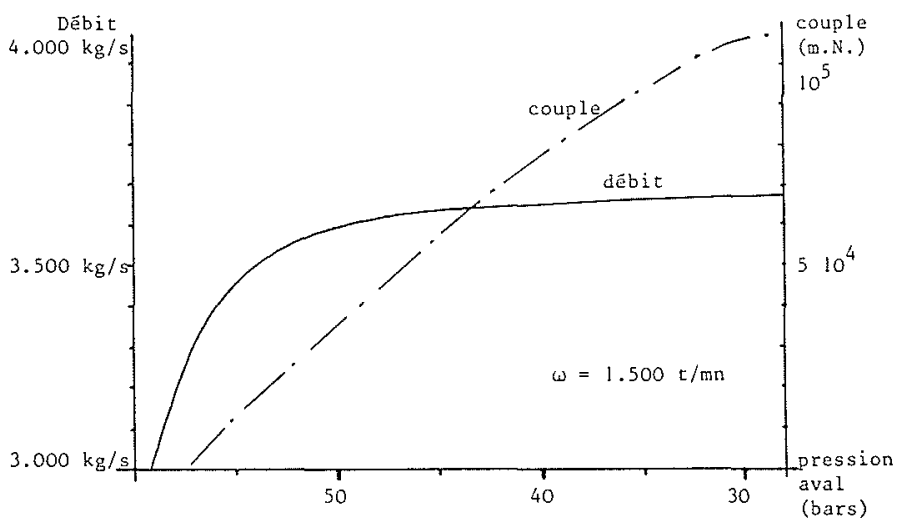

Figure 7 - Evolution du couple et du débit lors d'une ouverture de brèche aval.

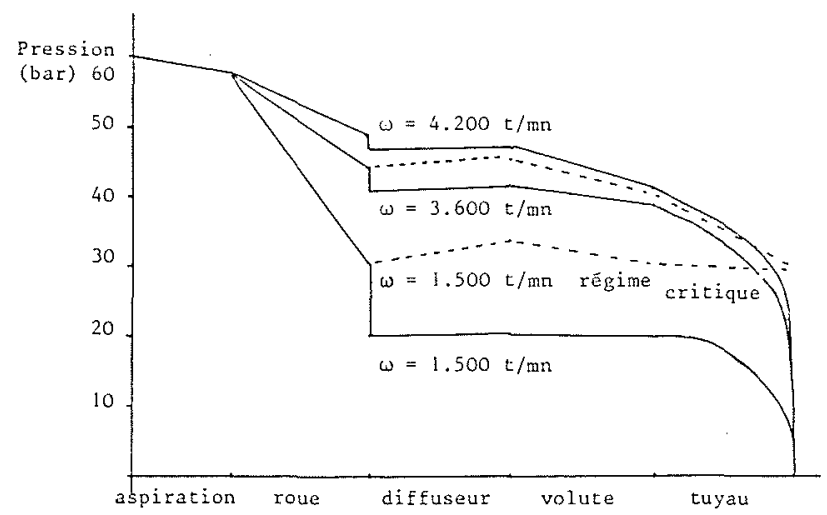

Figure 8 - brèche pleine ouverture. Profills de pression. 


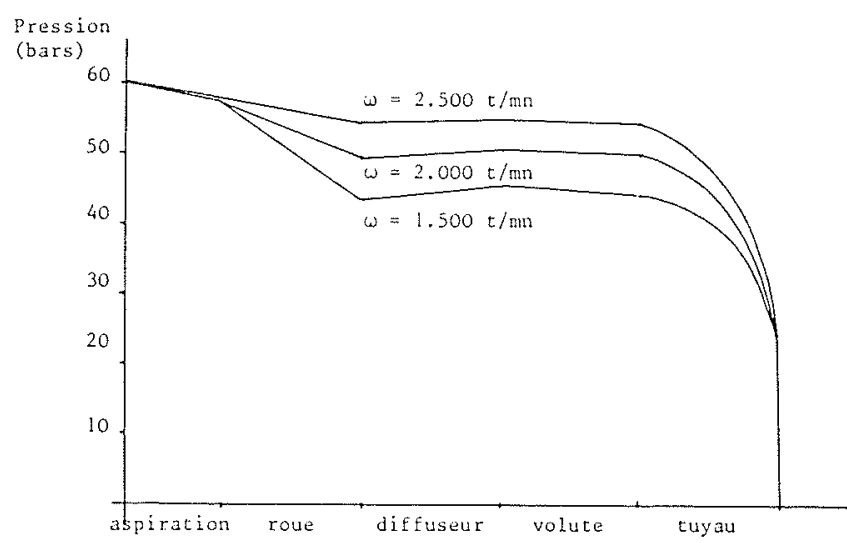

Figure 9 - brèche ouverture $50 \%$. Profils de pression.

c'est donc elle qui limitera le débit. Le couple initial est fortement réduit ( $\cong 2$ fois le couple nominal) et reste constant même si le $\Delta P$ aux bornes de la tuyauterie augmente. La vitesse de rotation de la pompe augmentera plus lentement. On observera sur la figure 9 la modification des profils de pression en fonction de la vitesse. Le couple devient nul pour une vitesse de rotation de l'ordre de $2400 \mathrm{tr} / \mathrm{min}$, avec un débit de $4550 \mathrm{~kg} / \mathrm{s}$.

\section{Conclusion}

L'étude du comportement d'une pompe en régime diphasique accidentel nous a conduits à développer une analyse théorique ainsi qu'une campagne expérimentale. Cette analyse a mis en évidence l'importance du transfert de masse entre phase (donc du retard thermodynamique) et du glissement interfacial dans la modélisation du comportement de la pompe. Nous avons donc utilisé le modèle d'écoulement diphasique dit "à deux fluides" développé par l'équipe mixte SEPTEN/CEA, que nous avons intégré à une description physique de l'écoulement dans la pompe. Ceci nous a permis de prendre en compte différents phénomènes (désadaptation de l'écoulement sur les aubage post-rotation) et de pouvoir ainsi décrire les principaux aspects du comportement de la pompe (débit critique, dégradation des courbes de hauteur) sans utilisation de corrélations supplémentaires.

Les comparaisons expérimentales effectuées d'une manière systématique sur les essais EPOPEE, ainsi que sur quelques points d'essais d'autres campagnes (essais EVA et essais Combustion Engineering), ont montré la bonne aptitude du modèle à représenter le comportement de la pompe en régime accidentel. Nous présentons donc ici un modèle un peu plus complexe que ceux utilisés précédemment, mais dont la transposition à l'échelle 1 est relativement sûre car elle représente bien les phénomènes physiques en faisant abstraction de corrélations propres aux essais de pompe.

L'application de ce modèle au calcul de survitesse montre qu'il est indispensable de représenter l'ensemble du système car le couplage entre les différents éléments est souvent très important.

\section{Références :}

[1] POCHARD R. - Etudes de sensibilité liées aux calculs d'accidents de référence des réacteurs de puissance. CEADSN - Rapport Searel n $n^{\circ} 79 / 03$.

[2] GRISON P. - LAURO J.F. - Modélisation d'une pompe pour un écoulement monophasique dans les trois premiers cuadrants. Rapport E.D.F. $\mathrm{n}^{\circ} \mathrm{HP} 316 / \mathrm{E} 02 / 80.01$.

[3] NIENALTOWSKA E. - Phénomènes dynamiques et thermiques pour l'ébullition et la cavitation à l'échelle de la bulle de vapeur. Thèse Ingénieur Docteur - Université Paris V. Novembre 1979.

[4] GRISSON P., LAURO J.F. - "EPOPEE" - Interpetation des essais "Veine inerte". Rapport E.D.F. $\mathrm{n}$ " $316 /$ E02/78.03.

[5] GRISON P., LAURO J.F. - Fonctionnement des pompes en écoulement diphasique eau-vapeur. ENTROPIE $\mathrm{n}^{\circ} 83-$ 1978.

[6] GRISON P., LAURO J.F. -- Experimental and theoretical investigations about two-phase critical flow through a pump Polyphase-flow in turbomachinery. ASME San Francisco 1978

[7] BOURE, FRITTE, GIOT and RECCREUX. - Highlights on two-phase critical flow. Int. J. Multiphase flow Vol. 3 - p. $1-22,1976$.

[8] PINET B. - Modélisation des écoulements pour l'analyse de l'accident de perte de réfrigérant primaire (APRP). ENTROPIE n $83-1978$.

[9] GRISON P., LAURO J.F. - EPOPEE - HEXPO, modèle de pompe en diphasique. Rapport E.D.F. $\mathrm{n}^{\circ}$ HP $316 / \mathrm{E} 02 /$ 82.04 .

[10] GRISON P. , LAURO J.F., DUBAIL A., MARSIGNE C. EPOPEE - Mesure du taux de vide à l'amont et à l'aval de la pompe - (Théorie - résultat analyse). Rapport E.D.F. $\mathrm{n}^{\mathrm{C}} \mathrm{HP} 316 / \mathrm{E} 02 / 81.01$.

[11] WILSON D.C. - Analytical models and experimental studies of centrifugal-pump performance in two phase flow. EPRI Report n ${ }^{\circ} \mathrm{NP} 677-1979$.

[12] ZARECHUAK, DAMERELL, CHAPIN, WU. - Thermodynamic model of centrifugal pump performance in twophase flow. Proc. of ANS - Octobre 1980.

[13] GRANDJEAN C. - Modèle Pompe Edipe. Rapport interne C.E.A. - DRE/SIT/SEMTH.

[14] GRISON P., LAURO J.F. - EPOPEE - Description générale de la Boucle d'Essais. Rapport E.D.F. n HP $316 /$ E02/82-01.

[15] LOUSTALOT J.P., LEPARC J. - EPOPEE - Fonctionnement de la boucle EPOPEE. Rapport E.D.F. $\mathrm{n}^{\circ}$ HP $316 /$ E01/80-01.

[16] GRISON P., LAURO J.F. - EPOPEE - Compte rendu des essais débit positif - Rotation positive. Rapport E.D.F. $n^{\circ} \mathrm{P} 316 / \mathrm{E} 02 / 79-01$.

[17] KUCIEL C. - Compte-rendu des essais EPOPEE - débit négatif - rotation positive et négative. Rapport E.D.F. $n^{\circ} \mathrm{P} 316 / \mathrm{E} 02 / 79-02$.

[18] GRISON P., LAURO J.F. -- Comportement des pompes en régime diphasique. La Houille Blanche n ${ }^{\circ} 6 / 7,1979$.

[19] GRISON P., LAURO J.F. - Compte-rendu des essais en eau froide d'une maquette au $1 / 10^{e}$ de pompe primaire Essais EPOPEE - Phase préliminaire) Rapport E.D.F. $\mathrm{n}^{\circ} \mathrm{P} 31 / \mathrm{E} 02 / 78-02$

[20] GRISON P., LAURO J.F. - EPOPEE - Comparaison des résultats d'essais et des calculs théoriques - $1^{\text {er }}$ quadrant. Rapport E.D.F. $n^{\circ}$ HP 316/E02/82-02.

[21] GRISON P., LAURO J.F. - EPOPEE - Comparaison des résultats d'essais et des calculs thériques $-3^{e}$ quadrant. Rapport E.D.F. n ${ }^{\circ}$ HP 316/E02/82-03.

[22] LAURO J.F. - Essais sur maquette du comportement d'une pompe PWR lors d'un $A D R$. Rapport E.D.F. $\mathrm{n}^{\circ} \mathrm{HJ}$ 31/D02/75-3. 


\section{Discussion}

Président : J. DODU

\section{M. le Président. - Je donne la parole à M. GRISON.}

M. GRISON. - J'ai quelque scrupule, après la modélisation tridimensionnelle qui vient d'être présentée de ne parler que de modélisation monodimensionnelle; je me sens un peu gêné. Ce n'est pas le schéma numérique qui est important, c'est la modélisation physique des phénomènes.

\section{Exposé de M. GRISON}

M. le Président. - Je remercie M. GRISON de son exposé et j'ouvre la discussion.

M. BONNIN. - Je voudrais apporter trois choses à M. GRISON : d'abord des compliments ensuite un commentaire et, pour finir, lui poser une question.

Je crois qu'il faut vraiment vous complimenter parce que vous avez mis sur pied quelque chose de très important, quand on pense aux installations que cela requiert.

En particulier, j'attire l'attention des auditeurs sur le nom qui a été donné au modèle: jamais un nom de code n'a été aussi bien mérité "EPOPEE"

Vous avez obtenu des résultats très intéressants dont tout le monde ne perçoit pas (peut-être) toutes les difficultés que représente l'expérimentation en diphasique à hautes pressions et températures sur une machine.

Vous vous souvenez certainement que, il y a quelques années, j'avais établi des règles de similitude sur une telle pompe. Cela n'étonnera personne de savoir qu'il était impossible de tou représenter, on ne savait pas si on pouvait prendre un phénomène de transfert au choix. Je note que les résultats d'essais ont montré, suivant les configurations, quel était le phénomène essentiel qui imposait sa loi à l'évolution des phénomènes physiques. Dans une étude théorique antérieure sur la similitude (que l'un des auteurs avait dû présenter à ma place dans un congrès), j’avais montré qu'il était nécessaire de limiter la représentation des transferts entre phases à un seul transfert pou éviter les incompatibilités entre les conditions de similitude. Les résultats obtenus permettent donc de valider une similitude dans chaque cas identifié.

Grâce aux travaux de M. GRISON on peut choisir la loi et la représenter effectivement avec la pompe modèle.

J'en arrive à ma question: pour le passage direct en diphasiques que vos essais sont les seuls à réaliser dans une turbomachine, avez-vous tenu compte de l'état de nucléation de l'eau et de ses variations possibles en similitude, et quelle est à votre avis l'importance de ce facteur?

M. GRISON. - Non, on n'a pas tenu compte de ces variations pour la bonne raison qu'on n'avait pas de mesures de cette nucléation. On a tenté, à un moment, de faire de telles mesures, mais, pour cela, il aurait fallu mesurer à une température de $300^{\circ} \mathrm{C}$ dans l'échantillon à une pression de 120 bars. Nous ne sommes pas parvenu à une mesure correcte; dans ces conditions, nous n'avons pas tenu compte de ce phénomène.
En outre, je ne pense pas que cela ait une importance considérable du fait que dans la pompe, quand on est à fort débit, on se trouve très vite très désadapté, avec des écoulements secondaires très importants un peu partout dans la pompe: En fait, c'est cette macroturbulence qui existe dans la pompe qui va générer tous les différents germes, ce qui fait que l'on aura un grossissement très rapide des bulles.

$M$. le Président. - En ce qui concerne la réalisation expérimentale, vous avez travaillé par rafales. Quelle est l'importance de l'instrumentation pour les rafales? On a parlé de 700 points de mesures.

M. GRISON, - Pour la boucle, on devait avoir une trentaine de capteurs de pression, deux mesures de taux de vide en amont et en aval de la pompe, plus des mesures classiques (couple, vitesse, poussée axiale) et tous Ies capteurs étaient explorés chaque $1 / 10^{e}$ de seconde.

La durée d'un point d'essai est de l'ordre de 20 secondes.

Les différentes marches qu'on a vues sur la courbe représentent, en gros, 20 secondes. Il y a 5 secondes de transitoires dues au fait que la régulation réagit. Toutes ces mesures ont été traitées directement sur calculateur, bien sûr.

Je voudrais ajouter quelque chose à la remarque de M. BONNIN. Il est effectivement important de voir que, suivant les différents domaines ò̀ l'on va se placer, les phénomènes physiques qui vont prédominer seront très différents. J'ai un peu effleuré ce point lors de mon exposé.

Quand on se place dans les conditions normales de fonctionnement d'une pompe, avec un débit pas trop fort, ce sont les phénomènes de glissement entre phases qui sont prédominants. Une courbe a été publiée par les Américains sur leurs essais; ils avaient toute latitude pour faire varier le taux de vide pour un débit relativement faible car ils procédaient par mélange d'eau et vapeur. Les Américains ont trouvé une courbe à peu près du même type que la courbe que j'avais montrée: hauteur diphasique/sur hauteur monophasique. Pour des débits relativement faibles, c'est ce phénomène qui est prépondérant. Il explique bien ce type de courbe qu'on ne savait pas du tout expliquer avant.

La dispersion des mesures est relativement grande, et on ne peut pas espérer, sur le plan théorique, arriver à une modélisation très poussée, très fine. Par contre, quand on va passer à des débits très forts, ce sont les régimes à débit critique qui vont imposer leur loi. Le glissement entre phase joue beaucoup moins.

M. HENR $Y$. - Avez-vous trouvé une bonne corrélation entre les essais et la modélisation sur la hauteur?

M. GRISON. - Pour être tout à fait honnête, je dirai que la corrélation est bonne dans le premier quadrant; c'est là où c'est le plus simple. Par contre, dans le deuxième quadrant, il y a une forte dissipation d'énergie et c'est beaucoup moins bon; dans certains cas, on arrive à des distorsions de l'ordre de $50 \%$; je crois que c'est dû aux difficultés expérimentales et théoriques. Quant au troisième quadrant, on trouve des accords acceptables. 